\title{
Bacteria-bacteriophage cycles facilitate Cholera outbreak cycles: an indirect Susceptible - Infected - Bacteria - Phage (iSIBP) model-based mathematical study
}

\author{
Asma Al Habees ${ }^{1}$, Eman Aldabbas ${ }^{1}$, Nicola L. Bragazzi ${ }^{2}$, and Jude \\ D. $\mathrm{Kong}^{2,3, *}$ \\ ${ }^{1}$ Department of Mathematics, The University of Jordan, Jordan \\ ${ }^{2}$ Department of Mathematics and Statistics, York University, \\ Toronto, ON M3J 1P3, Canada \\ ${ }^{3}$ Canadian Center for Diseases Modeling (CDM), York \\ University,Toronto, ON M3J 1P3, Canada \\ *Correspondence: jdkong@yorku.ca
}

February 15, 2021

\begin{abstract}
Cholera is an acute enteric infectious disease caused by the Gram-negative bacterium Vibrio Cholerae. Despite a huge body of research, the precise nature of its transmission dynamics has yet to be fully elucidated. Mathematical models can be useful to better understand how an infectious agent can spread and be properly controlled. We develop a compartmental model describing a Human population, a bacterial population as well as a phage population. We show that there might be eight equilibrium points; one of which is a disease free equilibrium point. We carry out numerical simulations and sensitivity analyses and we show that the presence of phage can reduce the number of infectious individuals. Moreover, we discuss the main implications in terms of public health management and control strategies.
\end{abstract}

Keywords: Cholera, Bacteriophage, phage, basic reproduction number. 


\section{Introduction}

Cholera is an acute enteric infectious disease caused by the Gram-negative bacterium Vibrio cholerae ( $V$. cholerae), which spreads by water or food contaminated with feces. It causes diarrhea, which can lead to dehydration that might lead to death within hours if it untreated (Finkelstein, 1996). Its effect is more dangerous among individuals who have insufficient access to safe water and proper sanitation; it is also more dramatic in areas where basic environmental infrastructures are disrupted or have been destroyed. The World Health Organization (WHO) estimated that there are from 1.3 to 4 million cholera cases and 21,000 to 143,000 deaths due to cholera yearly (WHO, 17 January 2019). However, in countries with adequate health care, cholera imposes a much less dramatic burden.

The precise nature of the cholera transmission dynamics is not clear yet, despite a huge body of research and the many efforts of the scientific community. For example, (Cash et al., 1974) discussed the response of men to infection with $V$. cholerae. In (Snow, 1855), Snow's communication model of cholera was proposed. (Islam et al., 1994) discussed the role of blue-green algae in maintaining endemicity and seasonality of cholera in Bangladesh. (Colwell et al. 1996) proposed that a viable but non-culturable $V$. cholerae $\mathrm{O} 1$ strain can revert to a cultivable state in the human intestine (Colwell et al., 1996), and in (Hartley et al., 2005), the authors discussed a critical element in the ability of $V$. cholerae to cause outbreaks and epidemics.

It is of paramount importance to understand how V.cholerae can transmit through men. This would enable researchers and public health authorities to monitor the evolution of the outbreak and help put into effect adequate intervention measures that limit the effects of cholera spreading.

Mathematical models of infection transmission dynamics are extremely useful in better understanding how an infectious agent can spread and how can be effectively managed and controlled. More specifically, mathematical modeling of cholera has a long history; through the decades, many models have been devised and proposed. However, few of them have incorporated indirect transmission of the disease. Among these, the major models that employ ordinary differential equations are those built upon improved and branched off versions of the Cappasso and Paveri-Fontana model (Capasso and Paveri-Fontana, 1979) and the Codeco model (Codeço, 2001). For example, these models include those proposed by (Hartley et al., 2005), (Jensen et al., 2006), (Joh et al., 2009), (Tian and Wang, 2011), (Mukandavire et al., 2011) and (Kong et al., 2014).

The model employed in this project is an extension of the work by (Kong et al., 2014), which considers the role of the bacterophage, host and pathogen on the infection's sustainability. The bacteria population is assumed to be divided into two subgroups: non-pathogenic and pathogenic strains. This assumption is due to the 
fact that the pili protein is produced only by pathogenic strains and is necessary to anchor the bacteria to the wall of the gastrointestinal tract, so that infection can develop. It is during this crucial process that the phage targets the pili protein. The pili and toxin genes are activated by the same bacterial switch, so that toxin is produced during bacterial colonization of the gastrointestinal tract. Noting that only virulent strains of $V$. cholerae can produce toxin, this biological process is insured thanks to the phage and the bacterium transfer CTX (a genetic sequence that produces the toxin) through a very specific mechanism, see (Lab, 1997).

In the present paper, we find and discuss eight potentially equilibria, one of which is a free equilibrium point. We discuss the major epidemiological properties of the model, including the basic reproduction number $\mathcal{R}_{0}$, we perform mathematical simulations and carry out sensitivity analysis to test the robustness of our proposed model.

\section{Model formulation}

Our model describes the cholera transmission taking into account host, pathogen and bacteriophage dynamics. Here, the phage population is divided into two groups: non-pathogenic and pathogenic strains. In the compartmental model presented, the host population follows a Susceptible-Infectious-Recovered (SIR) framework with $S+I+R=N$, where $N$ is the total population size, which is kept constant. The bacteria population is described by the compartments $B_{p}$ and $B_{n p}$, while $P$ represents the bacteriophage population. Observe that the interaction between bacteria and bacteriophage follows the well-known predator-prey relationship. Hence, we use Holling 1 predation term $\theta\left(B_{n p}, P\right)=\theta P B_{n p}$, where $\theta$ is a constant of proportionality, $\theta\left(B_{n p}, P\right)$ is the conversion rate from non-pathogenic strain to pathogenic strain, induced by phage. $K_{1}$ is the half saturation constant of predation (the bacteria level at which predation occurs at half the maximum rate). The populations' dynamics is represented by the following system of ordi- 
nary differential equations:

$$
\begin{aligned}
\dot{S} & =-\alpha\left(B_{p}\right) S-\mu S+\mu N+\eta R \\
\dot{I} & =\alpha\left(B_{p}\right) S-\mu I-\delta I \\
\dot{R} & =\delta I-\mu R-\eta R \\
\dot{B}_{p} & =r B_{p}\left(1-\frac{B_{p}+B_{n p}}{K}\right)-\gamma_{1} \frac{B_{p}}{K_{1}+B_{p}} P+\xi I+\theta\left(B_{n p}, P\right) \\
\dot{B}_{n p} & =r B_{n p}\left(1-\frac{B_{p}+B_{n p}}{K}\right)-\gamma_{2} \frac{B_{n p}}{K_{1}+B_{n p}} P+\xi I-\theta\left(B_{n p}, P\right) \\
\dot{P} & =\beta\left(\gamma_{1} \frac{B_{p}}{K_{1}+B_{p}}+\gamma_{2} \frac{B_{n p}}{K_{1}+B_{n p}}\right) P-d P+\phi \xi I
\end{aligned}
$$

$\alpha\left(B_{p}\right) S$ defines the incidence term, where $\alpha\left(B_{p}\right)$ is the pathogenic bacteria density-dependent component. The indirect part of the incident term is defined as:

$$
\alpha\left(B_{p}\right)= \begin{cases}0, & B_{p}<c \\ \frac{a\left(B_{p}-c\right)}{\left(B_{p}-c\right)+H}, & B_{p} \geq c\end{cases}
$$

Human contamination of the water supply through infected feces contributes to both bacteria and phage levels and is called 'shedding' and it depends on both bacteria shedding of the infected individuals (a biological quantity) and the level of sanitation in the environment (an environmental assessment). Bacteria and phage shedding rates may not be the same, so the rate for the bacteria is $\xi$ and that for the phage is $\xi \phi$, where $\phi$ is constant. The list of model parameters values and their description are seen in Table 1 . The ranges of the parameters values were expanded from (Cash et al., 1974) and (Jensen et al., 2006). For numerical simulations, the values in Table 1 are taken from the literature. 
Table 1: Model parameter values, their description and associated units

\begin{tabular}{|c|c|c|c|}
\hline Parameter & Description & Values & Unit \\
\hline $\mathrm{r}$ & $\begin{array}{l}\text { Maximum per } \\
\text { capita pathogen } \\
\text { growth efficiency }\end{array}$ & $0.3-14.3$ & $D a y^{-1}$ \\
\hline K & $\begin{array}{l}\text { Pathogen carry- } \\
\text { ing capacity }\end{array}$ & $10^{5}-10^{7}$ & Cell liter $^{-1}$ \\
\hline$\overline{\mathrm{H}}$ & $\begin{array}{l}\text { Half-saturation } \\
\text { pathogen den- } \\
\text { sity }\end{array}$ & $10^{6}-10^{8}$ & Cell liter $^{-1}$ \\
\hline $\bar{a}$ & $\begin{array}{l}\text { Maximum rate } \\
\text { of infection }\end{array}$ & 0.1 & $D a y^{-1}$ \\
\hline$\xi$ & $\begin{array}{l}\text { Pathogen shed } \\
\text { rate }\end{array}$ & $0-100$ & Cellliter $^{-1}$ Day $^{-1}$ \\
\hline$\mu$ & $\begin{array}{l}\text { Human recovery } \\
\text { rate }\end{array}$ & 0.1 & $D a y^{-1}$ \\
\hline $\mathrm{N}$ & Total population & $10^{6}$ & Persons \\
\hline $\mathrm{c}$ & MID & $10^{5}-10^{7}$ & Cellliter $^{-1}$ \\
\hline$\beta$ & Phage burst size & $80-100$ & Virions Day ${ }^{-1}$ \\
\hline$\gamma_{1}$ & $\begin{array}{l}\text { maximum per } \\
\text { capita phage } \\
\text { absorption rate }\end{array}$ & $0-0.025$ & CellVirion $^{-1}$ Day $^{-1}$ \\
\hline$\gamma_{2}$ & $\begin{array}{l}\text { maximum per } \\
\text { capita phage } \\
\text { absorption rate }\end{array}$ & $0-0.025$ & CellVirion $^{-1}$ Day $^{-1}$ \\
\hline $\mathrm{d}$ & phage death rate & $0.5-7.9$ & Virion $^{-1}$ Day $^{-1}$ \\
\hline$\phi$ & $\begin{array}{l}\text { Mean phage } \\
\text { shed rate }\end{array}$ & $10^{-6}-1$ & Virions cell ${ }^{-1}$ \\
\hline$K_{1}$ & $\begin{array}{l}\text { Half saturation } \\
\text { bacteria preda- } \\
\text { tion density }\end{array}$ & $<K$ & Cell liter $^{-1}$ \\
\hline
\end{tabular}

\section{Forward Invariance}

We would like to identify a forwardly invariant set in which solutions of system (1) will be bounded.

From the first equation of system (1) we see that $\dot{S}>0$ if $S=0$. Thus, $S(t)>0$ for $t>0$. From the second equation of system (1) we see that if $I=0$, then $\dot{I}=\alpha\left(B_{p}\right) S \geq 0$. Since $\alpha\left(B_{p}\right) \geq 0$ by definition, and $S(t)>0$ for $t>0$, we get that $\dot{I} \geq 0$. If $R=0$, then form the third equation of system (1), we get $\dot{R}=\delta I$, 
and hence $R(t) \geq 0$. As $S+I+R=N$, we must have $S, I, R \leq N$. Clearly, if $P=0$, then $\dot{P}=\phi \xi I \geq 0$, and hence $P(t) \geq 0$ for $t>0$.

If $B_{n p}=0$, then from equation (6) of system (1) we get that $\dot{B}_{n p}=\xi I$, thus $B_{n p} \geq$ 0 . Similarly, if $B_{p}=0$, then from equation (4) we have $\dot{B}_{p}=\xi I+\theta P B_{n p} \geq 0$, and hence $B_{p} \geq 0$.

To find an upper bound for $B_{n p}$ and $B_{p}$, let $B=B_{n p}+B_{p}$. Then

$$
\begin{aligned}
\dot{B} & =\dot{B}_{n p}+\dot{B}_{p} \\
& =r B\left(1-\frac{B}{K}\right)-\gamma_{1} \frac{B_{p}}{K_{1}+B_{p}} P-\gamma_{2} \frac{B_{n p}}{K_{1}+B_{n p}} P+2 \xi I \\
& \leq r B\left(1-\frac{B}{K}\right)+2 \xi N
\end{aligned}
$$

Set $F(B)=r B\left(1-\frac{B}{K}\right)+2 \xi N=\frac{-r}{K} B^{2}+r B+2 \xi N$. Now, If $B=0$, then $F(0)=2 \xi N$. Moreover, $F(B)=0$ has two real roots, namely

$$
B_{1}=\frac{K r+K \sqrt{r^{2}+8(r \xi N) / K}}{2 r}>0,
$$

and

$$
B_{2}=\frac{K r-K \sqrt{r^{2}+8(r \xi N) / K}}{2 r}<0 .
$$

Clearly, $B_{2}<1<B_{1}$.

The graph of $F(B)$ is shown in Figure 1. Since $B(t) \geq 0$ for $t>0$, we conclude that $0 \leq B(t) \leq B_{1}$ for $t>0$. Thus, $B(t)$ is bounded above.

Since $B=B_{n p}+B_{p}$, and both $B_{n p}$ and $B_{p}$ are non-negative, we get $B_{n p}, B_{p} \leq B_{1}$. 


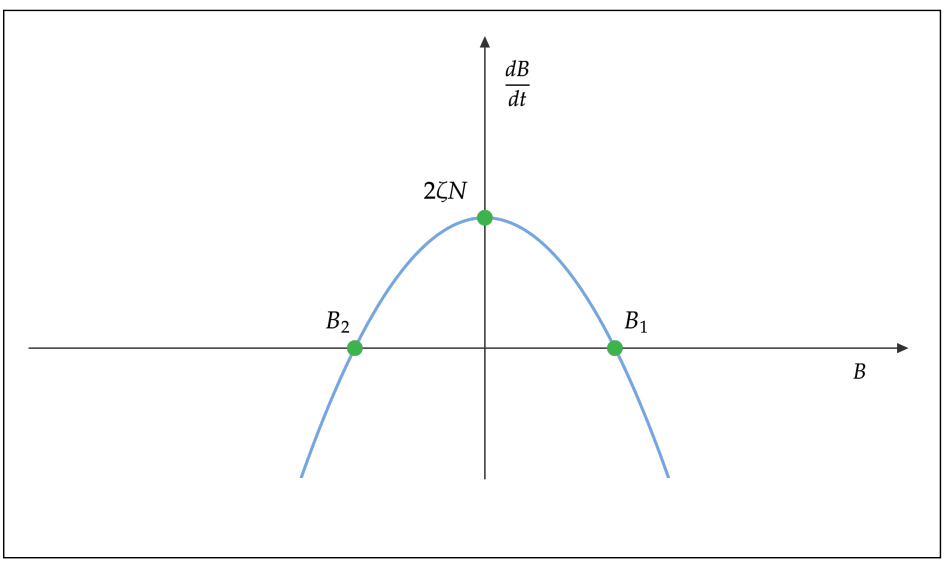

Figure 1: The graph of $F(B)=r B\left(1-\frac{B}{K}\right)+2 \xi N$

Finally, to find an upper bound for $P(t)$, the following lemma is needed.

Lemma 1. Define positive constants $u, \nu$ such that $\frac{((r+u) \beta)^{2} K}{4 r \beta}<\nu$. Then for all values of $B$, the following is true:

$$
0<\frac{r}{K} \beta B^{2}-((r+u) \beta) B+\nu .
$$

To show that $P(t)$ is bounded above, let $y(t)=\beta B+P$. Then

$$
\begin{aligned}
\frac{d y}{d t} & <r \beta B-\frac{r}{K} \beta B^{2}+2 \beta \xi N-d P \\
& <r \beta B-(r+u) \beta B+\nu-d P+2(\beta+\phi) \xi N
\end{aligned}
$$

Let $L=\min \{u, d\}$, and set $W=\nu+2(\beta+\phi) \xi N$. Then $\frac{d y}{d t}<-L y(t)+W$.

Now, by solving the ordinary differential equation $\dot{y}(t)=-L y(t)+W$, we get that $y(t)=\frac{W}{L}+c e^{-L t}$ for some constant $c$. Consequently, we get

$$
\limsup _{t \rightarrow \infty} y(t) \leq \frac{W}{L}
$$

We summarize the previous results is in the following proposition:

Proposition 1. Let $\mathbb{R}_{5}^{*}=\left\{\left(S, I, B_{p}, B_{n p}, P\right): S, I, B_{p}, B_{n p}, P \geq 0\right\}$. Then the set $\Omega=\left\{\left(S, I, B_{p}, B_{n p}, P\right) \in \mathbb{R}_{5}^{*}: S>0, S+I+R=N, \beta\left(B_{P}+B_{n p}\right)+P \leq \frac{W}{L}, B_{p}, B_{n p} \leq B_{1}\right\}$ 
defines a forward invariant region of system (1), where $W=2(\beta+\phi) \xi N+\nu$ and $L=\min \{u, d\}$, with $u, \nu>0$ are such $\frac{((r+u) \beta)^{2} K}{4 r \beta}<\nu$.

\section{Equilibria with no shedding and their stability}

In this section, we determine the equilibrium points of the model system when $\xi=0$, and then perform stability analysis of the equilibria.

\subsection{Existence of Equilibria}

Substituting $\xi=0$ in system (1) and noticing that $R=N-S-I$, the third equation is then not necessary. Thus, system (1) reduces to

$$
\begin{aligned}
\dot{S} & =-\alpha\left(B_{p}\right) S-\mu S-\eta S+\mu N+\eta N-\eta I \\
\dot{I} & =\alpha\left(B_{p}\right) S-\mu I-\delta I \\
\dot{B}_{p} & =r B_{p}\left(1-\frac{B_{p}+B_{n p}}{K}\right)-\gamma_{1} \frac{B_{p}}{K_{1}+B_{p}} P+\theta\left(B_{n p}, P\right) \\
\dot{B}_{n p} & =r B_{n p}\left(1-\frac{B_{p}+B_{n p}}{K}\right)-\gamma_{2} \frac{B_{n p}}{K_{1}+B_{n p}} P-\theta\left(B_{n p}, P\right) \\
\dot{P} & =\beta\left(\gamma_{1} \frac{B_{p}}{K_{1}+B_{p}}+\gamma_{2} \frac{B_{n p}}{K_{1}+B_{n p}}\right) P-d P
\end{aligned}
$$

To analyze the types of solutions that this model could produce, a steady state analysis was performed. The algebraic analysis is provided in Appendix A.

Case 1: If the pathogenic bacteria level is below or equal to the minimum infectious dose, then $\alpha\left(B_{p}\right)=0$. Hence system (2) becomes:

$$
\begin{aligned}
\dot{S} & =-\mu S-\eta S+\mu N+\eta N-\eta I \\
\dot{I} & =-\mu I-\delta I \\
\dot{B}_{p} & =r B_{p}\left(1-\frac{B_{p}+B_{n p}}{K}\right)-\gamma_{1} \frac{B_{p}}{K_{1}+B_{p}} P+\theta\left(B_{n p}, P\right) \\
\dot{B}_{n p} & =r B_{n p}\left(1-\frac{B_{p}+B_{n p}}{K}\right)-\gamma_{2} \frac{B_{n p}}{K_{1}+B_{n p}} P-\theta\left(B_{n p}, P\right) \\
\dot{P} & =\beta\left(\gamma_{1} \frac{B_{p}}{K_{1}+B_{p}}+\gamma_{2} \frac{B_{n p}}{K_{1}+B_{n p}}\right) P-d P
\end{aligned}
$$

Then system (3) has 4 disease-free equilibrium points which are listed below: 
1. $E_{0}=(N, 0,0,0,0)$ always exists.

2. $E_{1}=(N, 0, m, K-m, 0)$ always exists, where $m$ is a non-negative constant such that if $K \leq c$, then $m \leq K$, and if $K>c$, then $m \leq c$.

Special cases of $E_{1}$ are:

a. $E_{11}=(N, 0,0, K, 0)$.

b. $E_{12}=(N, 0, K, 0,0)$, where $K \leq c$.

3. $E_{2}=\left(N, 0, B_{p_{2}}, 0, P_{2}\right)$ exists if $\beta \gamma_{1}-d>0$. In this case, $B_{p_{2}}=\frac{d K_{1}}{\beta \gamma_{1}-d}>0$ is such that if $K \leq c$, then $B_{p_{2}} \leq K$, and if $K>c$, then $B_{p_{2}} \leq c$, so that $P_{2}=\frac{r}{\gamma_{1} K}\left(K_{1}+B_{p_{2}}\right)\left(K-B_{p_{2}}\right)>0$.

4. The interior point $E^{*}=\left(N, 0, B_{p}^{*}, B_{n p}^{*}, P^{*}\right)$, exits if the following conditions hold:

(i) $B_{p} *<c$.

(ii) $B_{p}^{*} \neq \frac{d K_{1}}{\beta \gamma_{1}-d}=B_{p_{2}}$. Note that the existence of $B_{p}^{*}$ and $B_{p_{2}}$ is contrary.

(iii) $B_{p}^{*} \neq \frac{K_{1}\left(d-\beta \gamma_{2}\right)}{\beta\left(\gamma_{1}+\gamma_{2}\right)-d}$.

(iv) $K>B_{p}^{*}+B_{n p}^{*}$.

(v) $B_{p}^{*}, B_{n p}^{*}$ and $P^{*}>0$.

Case 2: If the pathogenic bacteria level is above the minimum infectious dose, then $\alpha\left(B_{p}\right) \neq 0$, leaving us with the following system:

$$
\begin{aligned}
\dot{S} & =-\alpha\left(B_{p}\right) S-\mu S-\eta S+\mu N+\eta N-\eta I \\
\dot{I} & =\alpha\left(B_{p}\right) S-\mu I-\delta I \\
\dot{B}_{p} & =r B_{p}\left(1-\frac{B_{p}+B_{n p}}{K}\right)-\gamma_{1} \frac{B_{p}}{K_{1}+B_{p}} P+\theta B_{n p} P \\
\dot{B}_{n p} & =r B_{n p}\left(1-\frac{B_{p}+B_{n p}}{K}\right)-\gamma_{2} \frac{B_{n p}}{K_{1}+B_{n p}} P-\theta B_{n p} P \\
\dot{P} & =\beta\left(\gamma_{1} \frac{B_{p}}{K_{1}+B_{p}}+\gamma_{2} \frac{B_{n p}}{K_{1}+B_{n p}}\right) P-d P
\end{aligned}
$$

Let $\Gamma_{1}=a(\mu+\delta+\eta)\left(B_{p}-c\right)+(\mu+\eta)(\mu+\delta)\left(B_{p}-c+H\right)$. Then, one can easily check that $\dot{I}=0$ if $I_{1}=\frac{\alpha\left(B_{p}\right)}{\mu+\delta} S$, and hence $\dot{S}=0$ if $S_{1}=(\mu+\eta)(\mu+\delta)\left(\frac{\left(B_{P}-c\right)+H}{\Gamma_{1}}\right) N$. Consequently, 
$I_{1}=\left(\frac{a(\mu+\eta)\left(B_{p}-c\right)}{\Gamma_{1}}\right) N$.

Solving the last three equations of system (4), we get the following equilibrium points:

1. $E_{3}=\left(S_{1}, I_{1}, m, K-m, 0\right)$ exists if $K>c$.

In this case, $m$ is a positive constant such that if $K>c$, then $c<m \leq K$.

Special case of $E_{3}$ is $E_{31}=\left(S_{1}, I_{1}, K, 0,0\right)$, where $K>c$.

2. $E_{4}=\left(S_{1}, I_{1}, B_{p_{4}}, 0, P_{4}\right)$ exists if $K>c$ and $\beta \gamma_{1}-d>0$.

In this case, $B_{p_{4}}=B_{p_{2}}=\frac{d K_{1}}{\beta \gamma_{1}-d}>0$ is such $c<B_{p_{4}} \leq K$, so that $P_{4}=\frac{r}{\gamma_{1} K}\left(K_{1}+B_{p_{4}}\right)\left(K-B_{p_{4}}\right)>0$.

3. The interior point $E^{* *}=\left(S_{1}, I_{1}, B_{p}^{* *}, B_{n p}^{* *}, P^{* *}\right)$, exits if the following conditions are hold:

(i) $B_{p}^{* *}>c$.

(ii) $B_{p}^{* *} \neq \frac{d K_{1}}{\beta \gamma_{1}-d}=B_{p_{4}}$. Note that the existence of $B_{p}^{* *}$ and $B_{p_{4}}$ is contrary.

(iii) $B_{p}^{* *} \neq \frac{K_{1}\left(d-\beta \gamma_{2}\right)}{\beta\left(\gamma_{1}+\gamma_{2}\right)-d}$.

(iv) $K>B_{p}^{* *}+B_{n p}^{* *}$.

(v) $B_{p}^{* *}, B_{n p}^{* *}$ and $P^{* *}>0$.

\subsection{Stability of Equilibria}

Depending on the pathogenic bacteria level, the linearization of system (2) has two forms, one for system (3) when $\alpha\left(B_{p}\right)=0$, denoted by $J$, and one for system (4) when $\alpha\left(B_{p}\right) \neq 0$, denoted by $\hat{J}$.

The stability of the equilibria $E_{0}, E_{1}, E_{2}$ are stated in Theorem 1 and that of the equilibria $E_{3}, E_{4}$ are stated in Theorem 2 .

Let

$$
R_{B}=\frac{\beta}{d}\left(\frac{\gamma_{1} m}{K_{1}+m}+\frac{\gamma_{2}(K-m)}{K_{1}+K-m}\right)
$$

. Then, we have

Theorem 1. The equilibrium point $E_{0}$ is always unstable. The equilibrium point $E_{1}$ might be stable if $R_{B}<1$, and consequently the equilibrium point $E_{11}$ might be stable if $\frac{\beta \gamma_{2} K}{d\left(K_{1}+K\right)}<1$ and the equilibrium point $E_{12}$ might be stable if $\frac{\beta \gamma_{1} K}{d\left(K+K_{1}\right)}<1$. That is, $E_{12}$ might be unstable if $E_{2}$ exists. The equilibrium point $E_{2}$, if exists, is always unstable. 
Proof. Set: $U=r-\frac{2 r B_{p}}{K}-\frac{r B_{n p}}{K}-\frac{K_{1} \gamma_{1} P}{\left(K_{1}+B_{p}\right)^{2}}$ and $V=r-\frac{r B_{p}}{K}-\frac{2 r B_{n p}}{K}-\frac{K_{1} \gamma_{2} P}{\left(K_{1}+B_{n p}\right)^{2}}-\theta P$. Then the Jacobian matrix for model system (3) is given as follows:

$$
J=\left[\begin{array}{ccccc}
-(\mu+\eta) & 0 & 0 & 0 & 0 \\
0 & -(\mu+\delta) & 0 & 0 & 0 \\
0 & 0 & U & \frac{-r B_{P}}{K}+\theta P & \frac{-\gamma_{1} B_{P}}{K_{1}+B_{P}}+\theta B_{n p} \\
0 & 0 & -\frac{r B_{n p}}{K} & V & \frac{-\gamma_{2} B_{n p}}{K_{1}+B_{n p}}-\theta B_{n p} \\
0 & 0 & \frac{\beta \gamma_{1} K_{1} P}{\left(K_{1}+B_{P}\right)^{2}} & \frac{\beta \gamma_{2} K_{1} P}{\left(K_{1}+B_{n p}\right)^{2}} & \beta\left(\frac{\gamma_{1} B_{p}}{K_{1}+B_{p}}+\frac{\gamma_{2} B_{n p}}{K_{1}+B_{n p}}\right)-d
\end{array}\right]
$$

Let $J_{i}$ be the Jacobian matrix evaluated at $E_{i}, i \in\{0,1,2, *\}$.

From the Jacobian matrix $J_{0}$, it is found that its eigenvalues are $-(\mu+\eta),-(\mu+$ $\delta),-d<0$ and $r$. Since $r>0$, then $E_{0}$ is always unstable.

From the Jacobian matrix $J_{1}$, it is found that its eigenvalues are $-(\mu+\eta),-(\mu+$ $\delta),-r, 0$ and $\beta\left(\frac{\gamma_{1} m}{K_{1}+m}+\frac{\gamma_{2}(K-m)}{K_{1}+K-m}\right)-d$. Hence, $E_{1}$ might be stable if the following condition holds:

$$
R_{B}=\frac{\beta}{d}\left(\frac{\gamma_{1} m}{K_{1}+m}+\frac{\gamma_{2}(K-m)}{K_{1}+K-m}\right)<1 .
$$

Considering the special case $E_{11}$, we get the following eigenvalues: $-(\mu+\eta),-(\mu+$ $\delta),-r, 0$ and $\frac{\beta \gamma_{2} K}{K+K_{1}}-d$. Thus, if $\frac{\beta \gamma_{2} K}{d\left(K_{1}+K\right)}<1$, then $E_{11}$ might be stable, and if $\frac{\beta \gamma_{2} K}{d\left(K_{1}+K\right)}>1$, then $E_{11}$ might be unstable.

When considering the equilibrium point $E_{12}$, we found that the eigenvalues corresponding to $J_{1}$ are: $-(\mu+\eta),-(\mu+\delta),-r, 0$ and $\frac{\beta \gamma_{1} K}{K+K_{1}}-d$. So, $E_{12}$ might be stable if $\frac{\beta \gamma_{1} K}{d\left(K+K_{1}\right)}<1$, which is equivalent to say that $E_{12}$ might be stable if $K<\frac{d K_{1}}{\beta \gamma_{1}-d}=B_{p_{2}}$. But if $E_{2}$ exists, then $K>\frac{d K_{1}}{\beta \gamma_{1}-d}=B_{p_{2}}$ and hence, $E_{12}$ might be unstable whenever $E_{2}$ exists.

From the Jacobian matrix $J_{2}$, it is found that none of the eigenvalues is zero, and one of the eigenvalue is $\lambda=r-\frac{r B_{p_{2}}}{K}-\frac{\gamma_{2} P_{2}}{K_{1}}-\theta P_{2}$. Now, if $\lambda \leq 0$, then

$$
\begin{aligned}
K & \leq \frac{d K_{1}}{\beta \gamma_{1}-d}+\left(\frac{\gamma_{2}}{\gamma_{1} K_{1}}+\frac{\theta}{\gamma_{1}}\right)\left(K_{1}+\frac{d K_{1}}{\beta \gamma_{1}-d}\right)\left(K-\frac{d K_{1}}{\beta \gamma_{1}-d}\right) \\
\left(1-\frac{\gamma_{2} \beta+\theta K_{1} \beta}{\beta \gamma_{1}-d}\right) K & \leq \frac{d K_{1}}{\beta \gamma_{1}-d}-\frac{d K_{1} \beta\left(\gamma_{2}+\theta K_{1}\right)}{\left(\beta \gamma_{1}-d\right)^{2}} \\
K & \leq \frac{d K_{1}}{\beta \gamma_{1}-d}=B_{p_{2}}
\end{aligned}
$$


But in order for $P_{2}$ to be positive, we must have $K>B_{p_{2}}$. Thus, we conclude that $\lambda>0$, and hence $E_{2}$ is unstable whenever it exists.

Theorem 2. The equilibrium point $E_{3}$ might be stable if $R_{B}>1$, where the equilibrium point $E_{31}$ might be stable if $\frac{\beta \gamma_{1} K}{d\left(K_{1}+K\right)}<1$. The equilibrium point $E_{4}$, if exists, is always unstable.

Proof. Set: $U=r-\frac{2 r B_{p}}{K}-\frac{r B_{n p}}{K}-\frac{K_{1} \gamma_{1} P}{\left(K_{1}+B_{p}\right)^{2}}$ and $V=r-\frac{r B_{p}}{K}-\frac{2 r B_{n p}}{K}-\frac{K_{1} \gamma_{2} P}{\left(K_{1}+B_{n p}\right)^{2}}-\theta P$. Then the Jacobian matrix for model system (4) is given as follows:

$$
\hat{J}=\left[\begin{array}{ccccc}
-\alpha\left(B_{p}\right)-\mu-\eta & 0 & \frac{-a S_{1} H}{\left(B_{p}-c+H\right)^{2}} & 0 & 0 \\
\alpha\left(B_{p}\right) & -(\mu+\delta) & \frac{-a S_{1} H}{\left(B_{p}-c+H\right)^{2}} & 0 & 0 \\
0 & 0 & U & \frac{-r B_{P}}{K}+\theta P & \frac{-\gamma_{1} B_{P}}{K_{1}+B_{P}}+\theta B_{n p} \\
0 & 0 & -\frac{r B_{n p}}{K} & V & \frac{-\gamma_{2} B_{n p}}{K_{1}+B_{n p}}-\theta B_{n p} \\
0 & 0 & \frac{\beta \gamma_{1} K_{1} P}{\left(K_{1}+B_{P}\right)^{2}} & \frac{\beta \gamma_{2} K_{1} P}{\left(K_{1}+B_{n p}\right)^{2}} & \beta\left(\frac{\gamma_{1} B_{p}}{K_{1}+B_{p}}+\frac{\gamma_{2} B_{n p}}{K_{1}+B_{n p}}\right)-d
\end{array}\right]
$$

Let $\hat{J}_{i}$ be the Jacobian matrix evaluated at $E_{i},(i=3,4, * *)$.

From the Jacobian matrix $\hat{J}_{3}$, it is found that the eigenvalues are: $-\alpha(m)-$ $\mu-\eta,-(\mu+\delta),-r, 0$ and $\beta\left(\frac{\gamma_{1} m}{K_{1}+m}+\frac{\gamma_{2}(K-m)}{K_{1}+K-m}\right)-d$. Hence, $E_{3}$ might be stable if $R_{B}<1$.

Considering the equilibrium point $E_{31}=\left(S_{1}, I_{1}, K, 0,0\right), K>c$, we get the following eigenvalues: $-(\alpha(K)+\mu+\eta),-(\mu+\delta),-r, 0$ and $\frac{\beta \gamma_{1} K}{K_{1}+K}-d$. Hence, $E_{31}$ might be stable if $\frac{\beta \gamma_{1} K}{d\left(K_{1}+K\right)}<1$.

From the Jacobian matrix $\hat{J}_{4}$, it is found that none of the eigenvalues is zero and one of the eigenvalues is $\lambda=r-\frac{r B_{p_{4}}}{K}-\frac{\gamma_{2} P_{4}}{K_{1}}-\theta P_{4}$, which was shown in the proof of Theorem (1) to be positive. Thus, $E_{4}$, if exists, is unstable.

\section{Equilibria with shedding and their stability}

In this section, we determine the equilibrium points of the model system when $\xi \neq 0$, and then perform stability analysis of the equilibria. 


\subsection{Existence of equilibria}

Noting that as $R=N-S-I$ the third equation of system (1) is not necessary, leaving us with the following system:

$$
\begin{aligned}
\dot{S} & =-\alpha\left(B_{p}\right) S-\mu S+\mu N+\eta R \\
\dot{I} & =\alpha\left(B_{p}\right) S-\mu I-\delta I \\
\dot{B}_{p} & =r B_{p}\left(1-\frac{B_{p}+B_{n p}}{K}\right)-\gamma_{1} \frac{B_{p}}{K_{1}+B_{p}} P+\xi I+\theta\left(B_{n p}, P\right) \\
\dot{B}_{n p} & =r B_{n p}\left(1-\frac{B_{p}+B_{n p}}{K}\right)-\gamma_{2} \frac{B_{n p}}{K_{1}+B_{n p}} P+\xi I-\theta\left(B_{n p}, P\right) \\
\dot{P} & =\beta\left(\gamma_{1} \frac{B_{p}}{K_{1}+B_{p}}+\gamma_{2} \frac{B_{n p}}{K_{1}+B_{n p}}\right) P-d P+\phi \xi I
\end{aligned}
$$

If $\alpha\left(B_{p}\right)=0$, then we will have the same equilibrium points $E_{0}, E_{1}, E_{2}$ and $E^{*}$ as in section 4.1, with the same conditions.

If $B_{P}>c$, then $\alpha\left(B_{p}\right) \neq 0$, and hence at steady state system (5) reduces to:

$$
\begin{aligned}
& 0=-\alpha\left(B_{p}\right) S-\mu S-\eta S+\mu N+\eta N-\eta I \\
& 0=\alpha\left(B_{p}\right) S-\mu I-\delta I \\
& 0=r B_{p}\left(1-\frac{B_{p}+B_{n p}}{K}\right)-\gamma_{1} \frac{B_{p}}{K_{1}+B_{p}} P+\xi I+\theta B_{n p} P \\
& 0=r B_{n p}\left(1-\frac{B_{p}+B_{n p}}{K}\right)-\gamma_{2} \frac{B_{n p}}{K_{1}+B_{n p}} P+\xi I-\theta B_{n p} P \\
& 0=\beta\left(\gamma_{1} \frac{B_{p}}{K_{1}+B_{p}}+\gamma_{2} \frac{B_{n p}}{K_{1}+B_{n p}}\right) P-d P+\phi \xi I
\end{aligned}
$$

Then system (6) has one equilibrium point, namely $E^{* * *}=\left(S_{1}, I_{1}, B_{p}^{* * *}, B_{n p}^{* * *}, P^{* * *}\right)$ which exists if the following condition holds:

$$
\gamma_{1} \frac{B_{p}^{* * *}}{K_{1}+B_{p}^{* * *}}+\gamma_{2} \frac{B_{n p}^{* * *}}{K_{1}+B_{n p}^{* * *}}<\frac{d}{\beta}
$$

where $S_{1}=(\mu+\eta)(\mu+\delta) \frac{\left(B_{p}^{* * *}-c\right)+H}{\Gamma_{1}} N$, and $I_{1}=\left(\frac{a(\mu+\eta)\left(B_{p}^{* * *}-c\right)}{\Gamma_{1}}\right) N$, with $\Gamma_{1}=a(\mu+\delta+\eta)\left(B_{p}^{* * *}-c\right)+(\mu+\eta)(\mu+\delta)\left(B_{p}^{* * *}-c+H\right)$. $P^{* * *}=-\phi \xi I_{1}\left[\frac{\left(K_{1}+B_{p}^{* * *}\right)\left(K_{1}+B_{n p}^{* * *}\right)}{\Gamma_{2}}\right]$, where

$$
\Gamma_{2}=\beta \gamma_{1} B_{p}^{* * *}\left(K_{1}+B_{n p}^{* * *}\right)+\beta \gamma_{2} B_{n p}^{* * *}\left(K_{1}+B_{p}^{* * *}\right)-d\left(K_{1}+B_{p}^{* * *}\right)\left(K_{1}+B_{n p}^{* * *}\right) .
$$

The proof of the existence of $E^{* * *}$ is provided in Appendix A. 


\subsection{Stability of Equilibria $E^{*}, E^{* *}, E^{* * *}$}

For the equilibrium points $E^{*}, E^{* *}, E^{* * *}$ we lack exact expressions for the equilibrium quantities, and so the local stability is difficult to be found analytically.

\section{Sensitivity Analysis}

The objective of this section is to discuss the sensitivity of the peak value and time to model parameters. We assume that recovered patient immediately become susceptible. We equally assume that within the period of our studies, deaths and births are equal. Thus our system reduces to the following system

$$
\begin{aligned}
\dot{S} & =-\alpha\left(B_{p}\right) S+\delta I \\
\dot{I} & =\alpha\left(B_{p}\right) S-\delta I \\
\dot{B}_{p} & =r B_{p}\left(1-\frac{B_{p}+B_{n p}}{K}\right)-\gamma_{1} \frac{B_{p}}{K_{1}+B_{p}} P+\xi I+\theta\left(B_{n p}, P\right) \\
\dot{B}_{n p} & =r B_{n p}\left(1-\frac{B_{p}+B_{n p}}{K}\right)-\gamma_{2} \frac{B_{n p}}{K_{1}+B_{n p}} P+\xi I-\theta\left(B_{n p}, P\right) \\
\dot{P} & =\beta\left(\gamma_{1} \frac{B_{p}}{K_{1}+B_{p}}+\gamma_{2} \frac{B_{n p}}{K_{1}+B_{n p}}\right) P-d P+\phi \xi I
\end{aligned}
$$

Using the normalized forward sensitivity index, we study the sensitivity of the parameters of System (7) to cholera outbreak peak and peak time. The normalized forward sensitivity index:

$$
\text { S.I. }=\frac{\partial x^{*}}{\partial p} \frac{p}{x^{*}}
$$

where $x^{*}$ is the quantity being considered (peak value or peak time), and $p$ is a parameter which $x^{*}$ depends upon. Sensitivity indices can be positive or negative which indicates the nature of the relationship, and it is the magnitude that ranks the strength of the relationship as compared to the other parameters. Because we do not have have an explicit formula for the quantities we are interested in (outbreak peak, outbreak peak time and the endemic steady state), we estimate $\frac{\partial x^{*}}{\partial p}$ using the central difference approximation:

$$
\frac{\partial x^{*}}{\partial p}=\frac{x^{*}(p+\Delta p)-x^{*}(p-\Delta p)}{2 \Delta p}+\mathcal{O}\left(\Delta p^{2}\right) .
$$

We choose $\Delta p=1 \%$ of $\mathrm{p}$.

plugging all these in (8) we get

$$
S . I=\frac{x^{*}(1.01 p)-x^{*}(0.99 p)}{0.02 x^{*}}
$$


Figure (2) contains the sensitivity of the outbreak peak (Figure (2a)) and the outbreak peak time (Figure (2b)) to the parameters of the model.

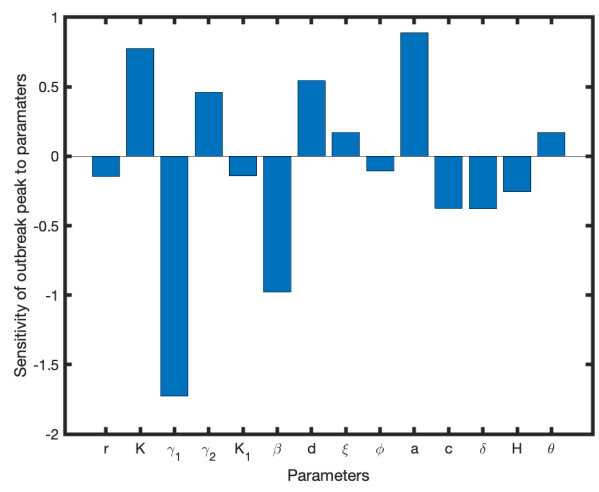

(a) The sensitivity of the outbreak peak to the parameters.

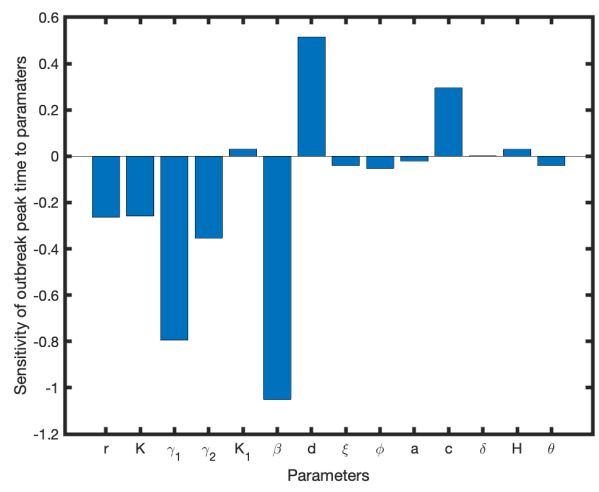

(b) The sensitivity of the outbreak peak time to the parameters

Figure 2: The sensitivity of the magnitude of outbreak peak and peak time to the parameters.

Figure (3) shows reservoir related parameters: phage attack rate and phage burst size have the strongest relationships to the magnitude of the outbreak peak and outbreak peak time. The Figure shows that if the phage attack rate and burst sizes are large, the magnitude of the outbreak peak value will be small and the time at which it occurs will be large. This indicates that phage-bacteria predator prey like cycles may have a key role to play in driving cholera outbreak cycles. Looking at the two parameters that can be controlled by policy-makers, these are $K$ and $\xi$. The carrying capacity $K$ has a large influence on the dynamics of the system. It has one of the largest sensitivity indices, being many times greater than that of the shedding rate $\xi$. Figure (3a) the sensitivity indices of the outbreak to these two important parameters. The Figure shows that the outbreak peak value increase as $K$ but almost has no response to the variation in $\xi$. This further emphasizes the importance of paying attention to the dynamics in the reservoir in order to control and prevent outbreaks. The figure shows that if there are good sanitation practices that prevent shedding, but without taking care of the dynamics in the reservoir the bacteria-phage dynamics may still be able to cause outbreak in human population. This is clearly illustrated in Figure (3b). In Figure (3b) we plot the time course dynamics of infected population (the right y-axis) and pathogenic bacteria population (left y-axis). The parameters values are chosen in such a way that we have two outbreaks every year. In such a situation, the figure illustrates that the bacteria population peaks before the human population. This 
indicates that the bacteria-bacteriophage cycles in the reservoir may be driving the outbreak cycles in the human population.

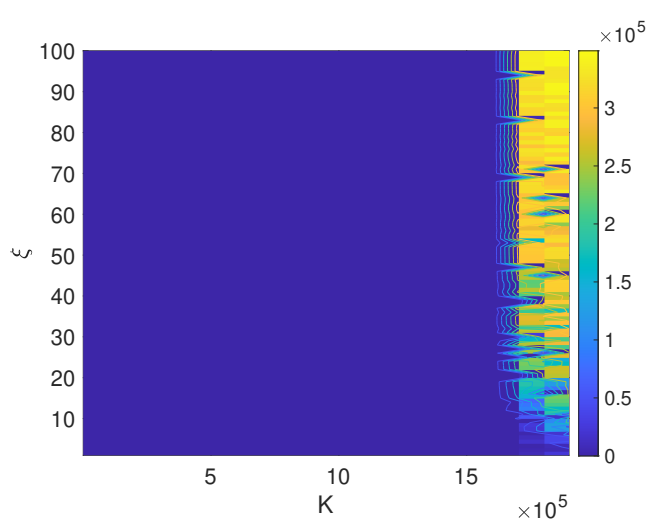

(a) Sensitivity of the out break peak to $\mathrm{K}$ and $\xi$. K varies from 100 to $2 \mathrm{e} 6$ and $\xi$ varies from 1 to 100 .

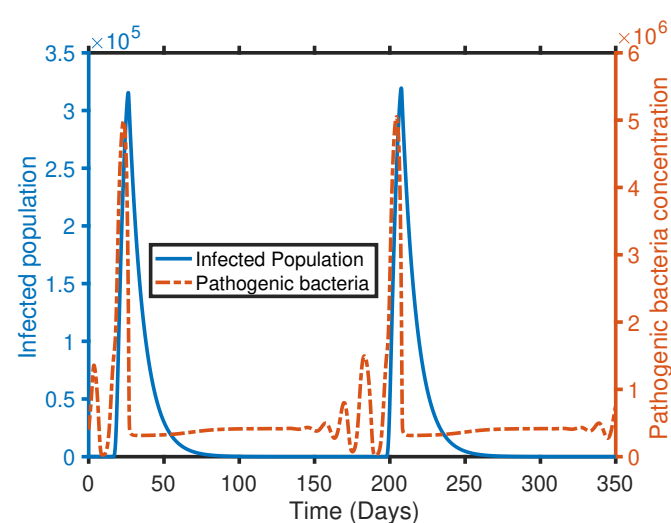

(b) Time course dynamics of the infected population and pathogenic phage levels in the reservoir

Figure 3: Microscopic cycles in the reservoir drives the macroscopic cycles in human population

Our finding that the bacteria-bacteriophage cycles in the reservoir may be driving the outbreak cycles in the human population has important practical implications in terms of public health policies. Our model confirms and expands empirical and theoretical proofs that lytic bacteriophages are one of the major drivers of cholera infection, shedding light on the complex interplay between the host, pathogen and phages (Nelson et al., 2009).

Cholera is one of the infectious diseases for which ecological factors and indirect transmission routes play a major role, together with those infections caused by pathogens like rotavirus, hantavirus, Legionella, Schistosoma, Cryptosporidium or Giardia. Devising mathematical models which incorporate bacteriophages can shed light on the epidemiological features of cholera and provide a better understanding of it. The seasonal self-limiting pattern of cholera epidemics can be explained taking into account the phage predation behavior of the $V$. cholerae during the late stages of the outbreak, which are usually characterized by significant phage shedding in stools. Bacteriophages are able to tune/modulate the bacterial infectivity and, as such, to lead to the collapse/quenching of the outbreak, with a very low probability of a phage-resistant variant to take-over (Silva-Valenzuela and Camilli, 2019). Advancements in the understanding of phages/bacteria interactions could potentially lead to the development of tools for monitoring and even predicting (forecasting/now-casting) of cholera outbreaks. For instance, it has been suggested that phage monitoring in acquatic environments and reser- 
voirs could be a feasible strategy for controlling and mitigating against the burden imposed by cholera (Silva-Valenzuela and Camilli, 2019).

Moreover, recent discoveries have shown that cocktails based on a number of virulent bacteriophages can effectively prevent $V$. cholerae infection (Yen et al., 2017). Phage therapy appears to be a promising pharmacological tool to counteract cholera transmission, given the organizational difficulties to implement mass vaccination campaigns in low-resource contexts as well as the lack of drugs and the issue imposed by multi-drug resistance (Bhandare et al., 2019), (Miller, 2003). In conclusion, mathematical models could play a significant role in elucidating the mechanisms of such a therapy, helping physicians optimize it (Jensen et al., 2006). As such, further research is warranted.

\section{Conclusion}

Cholera is an acute enteric infectious disease which imposes a relevant societal and clinical burden in developing countries, characterized by inadequate access to water, sanitation and lacking of proper healthcare facilities. Despite a huge body of research, the precise nature of cholera transmission dynamics has yet to be fully elucidated. Mathematical models can be useful to better understand how an infectious agent can spread and be properly controlled. In the present paper, we developed a compartmental model describing a human population, a bacterial population as well as a phage population. We showed that there might be eight equilibrium points; one of which is a disease free equilibrium point. We carried out extensive numerical simulations and sensitivity analyses and we showed that the presence of phages can significantly reduce the number of infectious individuals. Moreover, we discussed the main implications in terms of public health management and control strategies. Our proposed model can inform public health policies in low-resource settings, helping health policy- and decision-makers implemented evidence-informed programs aimed at targeting cholera outbreaks and epidemics. Moreover, our model can be further expanded and refined, inspiring further research in the field.

\section{Bibliography}

Cholera. https://www.who.int/news-room/fact-sheets/detail/cholera, 17 January 2019.

Bacteriophage Makes Vibrio Cholerae Deadly. Lab Med, 28(1):8-9, 1997. ISSN 0007-5027. 
Sudhakar Bhandare, Joan Colom, Abiyad Baig, Jenny M Ritchie, Habib Bukhari, Muhammad A Shah, Banwarilal L Sarkar, Jingliang Su, Brendan Wren, Paul Barrow, et al. Reviving phage therapy for the treatment of cholera. The Journal of infectious diseases, 219(5):786-794, 2019.

V Capasso and SL Paveri-Fontana. A mathematical model for the 1973 cholera epidemic in the european mediterranean region. Revue d'épidémiologie et de Santé Publiqué, 27(2):121-132, 1979.

Richard A. Cash, Stanley I. Music, Joseph P. Libonati, Merrill J. Snyder, Richard P. Wenzel, and Richard B. Hornick. Response of Man to Infection with Vibrio cholerae. I. Clinical, Serologic, and Bacteriologic Responses to a Known Inoculum. J Infect Dis, 129(1):45-52, 1974. ISSN 0022-1899. doi: 10.1093/infdis/129.1.45.

Cláudia Torres Codeço. Endemic and epidemic dynamics of cholera: the role of the aquatic reservoir. BMC Infect Dis, 1(1):1, 2001. ISSN 1471-2334. doi: 10.1186/1471-2334-1-1.

R. R. Colwell, P. Brayton, D. Herrington, B. Tall, A. Huq, and M. M. Levine. Viable but non-culturable Vibrio cholerae O1 revert to a cultivable state in the human intestine. World J Microbiol Biotechnol, 12(1):28-31, 1996. ISSN 1573-0972. doi: 10.1007/BF00327795.

Richard A. Finkelstein. Cholera, Vibrio cholerae O1 and O139, and Other Pathogenic Vibrios. In Samuel Baron, editor, Medical Microbiology. University of Texas Medical Branch at Galveston, Galveston (TX), 4th edition, 1996. ISBN 978-0-9631172-1-2.

David M. Hartley, J. Glenn Morris Jr, and David L. Smith. Hyperinfectivity: A Critical Element in the Ability of V. cholerae to Cause Epidemics? PLOS Medicine, 3(1):e7, 2005. ISSN 1549-1676. doi: 10.1371/journal.pmed.0030007.

Mohammad Sirajul Islam, Bohumil S Drasar, and R Bradley Sack. Probable Role of Blue-green Algae in Maintaining Endemicity and Seasonality of Cholera in Bangladesh: a Hypothesis. Journal of Diarrhoeal Diseases Research, 12(4):245256, 1994. ISSN 0253-8768.

Mark A. Jensen, Shah M. Faruque, John J. Mekalanos, and Bruce R. Levin. Modeling the role of bacteriophage in the control of cholera outbreaks. PNAS, 103(12): 4652-4657, 2006. ISSN 0027-8424, 1091-6490. doi: 10.1073/pnas.0600166103. 
Richard I. Joh, Hao Wang, Howard Weiss, and Joshua S. Weitz. Dynamics of Indirectly Transmitted Infectious Diseases with Immunological Threshold. Bull. Math. Biol., 71(4):845-862, 2009. ISSN 1522-9602.

Jude D. Kong, William Davis, and Hao Wang. Dynamics of a Cholera Transmission Model with Immunological Threshold and Natural Phage Control in Reservoir. Bull Math Biol, 76(8):2025-2051, 2014. ISSN 1522-9602.

Jeff F Miller. Bacteriophage and the evolution of epidemic cholera. Infection and immunity, 71(6):2981-2982, 2003.

Zindoga Mukandavire, Shu Liao, Jin Wang, Holly Gaff, David L. Smith, and J. Glenn Morris. Estimating the reproductive numbers for the 2008-2009 cholera outbreaks in Zimbabwe. Proceedings of the National Academy of Sciences, 108 (21):8767-8772, 2011.

Eric J Nelson, Jason B Harris, J Glenn Morris, Stephen B Calderwood, and Andrew Camilli. Cholera transmission: the host, pathogen and bacteriophage dynamic. Nature Reviews Microbiology, 7(10):693-702, 2009.

Cecilia A. Silva-Valenzuela and Andrew Camilli. Niche adaptation limits bacteriophage predation of vibrio cholerae in a nutrient-poor aquatic environment. Proceedings of the National Academy of Sciences, 116(5):1627-1632, 2019. ISSN 0027-8424. doi: 10.1073/pnas.1810138116. URL https ://www .pnas .org/ content/116/5/1627.

J. Snow. On the Mode of Communication of Cholera. John Churchill, London, 1855.

Jianjun Paul Tian and Jin Wang. Global stability for cholera epidemic models. Mathematical Biosciences, 232(1):31-41, 2011. ISSN 0025-5564. doi: 10.1016/j. mbs.2011.04.001.

Minmin Yen, Lynne S Cairns, and Andrew Camilli. A cocktail of three virulent bacteriophages prevents vibrio cholerae infection in animal models. Nature communications, 8(1):1-7, 2017. 